\title{
RUMORES EN LA ENSEÑANZA, PROFESORES DE FORMACIÓN PROFESIONAL
}

\author{
RUMOURS IN EDUCATION CIRCLES: VOCATIONAL TRAINING \\ TEACHERS
}

\author{
Francisco José Ramiro de la Mata \\ Universidad Rey Juan Carlos, Madrid. España/Spain \\ pacoctubre@,hotmail.com
}

Recibido/Received: 10/07/2012

Modificado/Modified: 17/07/2012

Aceptado/Accepted: 27/09/2012

\section{RESUMEN}

El presente trabajo es fruto del estudio sobre la comunicación informal por parte de los docentes en los centros públicos de la Comunidad de Madrid, dirigido en concreto hacia los profesores Técnicos de Formación Profesional y la zona sur de la citada comunidad. Las noticias publicadas en medios oficiales relacionadas con los exámenes de oposiciones y todo lo concerniente (amagos y afirmaciones) con los cambios en la enseñanza han servido para abonar un cultivo en el que crecen, entre individuos y/o grupos, comentarios, ciertos o no, pero que influyen en el clima de trabajo de los profesionales del campo de la docencia. Entre otras cuestiones relacionadas con los rumores se tratan el origen, lugar donde se producen, momento, forma, fiabilidad de los mismos y su influencia en el estado de ánimo.

\section{PALABRAS CLAVE}

Comunicación, docente, trabajo, ciclos formativos, motivación.

\section{SUMARIO}

1. Introducción. 2. Objetivos. 3. Fecha de investigación y contexto. 4. Los profesores técnicos de Formación Profesional. 5. Instrumentos utilizados en el estudio. 6. Análisis e interpretación de los resultados. 7. Conclusión. Bibliografía.

\begin{abstract}
This essay is the result of a research about informal communication amongst teachers in public schools of Madrid Autonomous Region. It is mainly focussed in Vocational Training teachers and in the southern area of the region. Data obtained in this research are intended to be used as a basis for a thesis. News published on official media related to competitive examinations and all what refers to changes in education (hints and statements) have acted as a breeding ground where different comments, either true or untrue, spread. All these have an influence on the working atmosphere for teaching professionals. Several issues regarding rumours are addressed, such as their source, the place where they occur, their timing, their form, their reliability, or the way they affect teachers' mood.
\end{abstract}

\section{KEYWORDS}

Communication, teachers, essay, training cycles, motivation. 


\section{CONTENTS}

1. Introduction. 2. Objectives. 3. Dates and context of the research. 4. Technical Teachers of Vocational Training. 5. Research tools. 6. Results analysis and interpretation. 7. Conclusion. References.

\section{INTRODUCCIÓN}

Este estudio pretende investigar una temática importante dentro de la comunicación como son los rumores en un sector, la enseñanza, donde las expectativas, el ambiente y los últimos cambios han proliferado en la divulgación de los mismos. El universo analizado son los profesores técnicos de Formación Profesional de la Comunidad de Madrid, ésta se encuentra dividida en 5 Direcciones de Área Territorial (DAT) de las que se ha elegido la DAT Sur.

Si la comunicación no verbal (que recibe también otros nombres como: "lenguaje mudo", "lenguaje del cuerpo", "lenguaje sin palabras", etc.) transmitida a través de los movimientos del cuerpo, la expresividad de la cara, la forma de ocupar el espacio, indumentaria, etc. (Fernández, 2003:53) es vital para la comunicación formal, más aun para la informal donde se elige el "cara a cara". La comunicación oral es una vía, subestimada a veces por estar al alcance de todos, para lograr objetivos, pero se puede convertir en contraproducente si el uso no es el adecuado dentro y fuera de la organización (O'C Leggett, 1993:24). Sabiamente empleado, hasta el más simple de los silencios puede aportar un rico recurso (Uña, 1994:199) y emocionar a quien es testigo de ello como para originar otro mensaje. A través de distintas redes sociales se producen un intercambio de estas emociones una vez que se abre la puerta de la confianza en un espacio de apoyo, produciéndose un proceso de socialización que beneficia al grupo y al individuo (Gallego, 2011:120). En un mundo donde la información se puede transmitir de manera casi instantánea a cuando se produce, se busca un equilibrio entre la información y la "exclusiva" (García y Díaz, 2010:314) esta exclusiva la encontramos muchas veces en pasillos y antesalas de las noticias formales por medio de los rumores.

Sucede con las redes sociales (entendidas como grupo de personas no como herramienta de comunicación) que al estar compuestas únicamente o bien por individuos donde todos se conocen con mucha anterioridad o por otro lado todos se desconocen, no son muy fructíferas en cuanto a intercambio de nuevos datos y si lo son, sin embargo, aquellas en los que se mezclan ambas características (Gallego, 2011:114). Lo mismo ocurre con la aportabilidad de los rumores que necesitan de la creatividad de la red, refiriéndonos a la composición de sus miembros.

Fuera de valoraciones positivas o negativas podemos aludir al rumor en las organizaciones como la forma de transmitir información a través de canales, la mayoría de las veces improvisados, como resultado de una interacción humana (Bel Mallén, 2005:144). Tradicionalmente, era llevado a través del vehículo de la palabra, hoy en día sigue ocurriendo, pero se le suman los medios cercanos a las nuevas tecnologías, dado el gran uso y el carácter informal del e-mail o las redes sociales (anudadas con herramientas informáticas), éstos suelen ser métodos no sólo para difundir rumores, sino también una forma para acelerar su transmisión y hacerlo a mayor número de personas. Conviene distinguir entre aquel, que en ocasiones precede a la información formal e incluso la 
amplía y, el chismorreo que se cuenta más en secreto y en el que suele salir alguien perjudicado.

En determinados momentos los mensajes no regulados por la comunicación formal parecen tener connotaciones negativas debido a la falta de credibilidad (merecida o no) de sus contenidos. Pero debemos considerar que, desde el punto de vista de la organización tiene dos visiones positivas: por un lado es una herramienta informativa al servicio de quién pueda manipularla; es difícil, en algunos casos, acabar con un rumor, pero es menos complicado generar uno nuevo (incluso que ponga en duda el primero); por otro lado, se convierte en "válvula de escape" de un amplio sector de la plantilla. Se transmite a través de canales informales y normalmente no va dirigido a personas específicas, (Martínez de Velasco y Nosnik, 2006:64), pese a ello no conviene subestimar su poder y utilidad dentro de la vida organizativa. La red en la que se transmite se caracteriza por divulgarse en más de un canal (contrariamente a lo que ocurre a veces con algunas comunicaciones formales) y a todos los niveles de jerarquía, o incluso ajenos a la organización (Fernández Collado, 2003:186).

Se convierte en asunto complicado detectar cuál es el verdadero momento del origen de una noticia de carácter informal en la mayoría de las ocasiones, tanto o más que quién o quiénes son los responsables del arranque o comienzo en una organización, pero sí podemos señalar y analizar cuáles son las causas a nivel general de su creación, éstas son: el interés por una situación, falta de información, ambigüedad, lo incierto y/o lo confuso, falta de motivación e inseguridad en el ámbito laboral o conflictos entre departamentos (Martínez de Velasco y Nosnik, 2006:65) (Bel Mallén, 2005:145). Mediante los rumores no solo se explican algunos hechos, independientemente de su veracidad, sino que en ocasiones se consigue que las cosas sucedan. Si se disemina el dato de que se va a concentrar al personal de un centro de trabajo en un lugar concreto, aunque no sea originalmente cierto, parte del personal estará allí (Burke, 2011:18).

Durante bastantes años el rumor ha sido criticado, asociándolo con la pérdida de tiempo y el cotilleo. Señalado en muchas ocasiones como producto de la envidia y con muy poco grado de veracidad, su origen es muy difícil de detectar pero es fácil reconocer la existencia de determinadas personas claves en su difusión (Lucas, 1997:186). Esta mala fama de los rumores se debe, entre otras causas, a que en canales formales, como la prensa se aprecia que de forma más clara se muestra un interés superior por desmentirlos que por confirmarlos (Mazo, 1996:129). Podemos puntualizar que la conversación es una herramienta al alcance de todos, aunque no siempre bien rentabilizada, pero importante en cualquier caso, con la que transmitimos sentimiento, indicamos lo que se piensa, lo que se hace y, apuntando hacia una organización, permite evitar y solucionar problemas además de crear oportunidades (Barker, 2001:21).

Existen medios para mejorar la motivación y con ella la productividad, para ello utilizamos como herramienta la comunicación, pero ¿cómo podemos motivar la comunicación? o dicho de otra manera ¿cómo mejoramos la comunicación? mediante empatía, simplificación del lenguaje, escuchar con atención, seguimiento del mensaje (cerciorarse de que ha sido recibido e interpretado correctamente), la retroalimentación y regulando la cantidad de información (Saavedra, 1998:98-100). El acortamiento de algunas de estas mejoras podría generar la existencia de rumores. Podemos hallar coincidencias entre preparar una buena negociación y un buen discurso (en ambos se trata de comunicar), sin embargo, las claves de la negociación están compuestas de una serie de elementos: ensayo, conocer las posiciones, hacer intercambios (regateo) y cumplir con los acuerdos 
(Oliver, 2005:26-32). Cabe la posibilidad de usar los rumores como herramienta para probar reacciones (por parte de la organización) o como intercambio de información (entre los miembros de esa organización) como estrategia planificada o parte de un trato. En períodos de crisis, tanto internas como generales, la comunicación informal denota cambios, por un lado se incrementan el número de rumores, y por otro existe un pesimismo generalizado incluso en las conversaciones informales ajenas al trabajo. Varios rumores sobre una misma temática consiguen aumentar el tiempo que se habla sobre ellos en cuantía mayor que si se sumaran estos por separado.

\section{OBJETIVOS}

Del entorno laboral por un lado y de la comunicación por otro, se desglosa la enseñanza (Profesores técnicos de FP) y la comunicación informal (los rumores), ambos son temas actuales a los que la ciencia sociológica aporta herramientas con las que poder estudiarlos e intentar lograr los siguientes objetivos:

- Conocer directamente la opinión de los profesores técnicos de FP en base al tema estudiado.

- Analizar el fenómeno del rumor y su influencia en el medio laboral de la enseñanza.

\section{FECHA DE INVESTIGACIÓN Y CONTEXTO}

La investigación ha tenido lugar durante los cursos académicos 2010-11 y 2011-12, aunque la principal extracción de datos se obtuvo del último; se han realizado cuestionarios a los profesionales de los centros educativos en noviembre de 2011 contrastado la información con entrevistas en profundidad en los meses de febrero y marzo de 2012.

Los exámenes de oposición se han venido desarrollando durante años, para primaria en año impar y para secundaria junto a ciclos formativos de FP en año par, por efectos prácticos una vez acabados los cursos a partir de junio. Durante otros años pasados ocupaban gran espacio, en las conversaciones espontáneas, todo lo referente a estas pruebas; a finales del 2011 y principios del 2012 la temática se volcó en los cambios y expectativas de la enseñanza. Cualquier especie vegetal modifica su estado con los cambios producidos por el paso del tiempo y es marcada por los periodos que definen las estaciones, a ello se suma la climatología que existe en cada momento, independientemente de que sea primavera o invierno; algo parecido ha ocurrido con los rumores en la enseñanza, marcados estacionalmente por fechas puntuales como han sido: comienzo del curso, fecha de exámenes de oposiciones, cambios de gobierno, aprobación de reformas etc. influenciadas a su vez por una climatología, un entorno, un ambiente de crisis económica en la que interinos de la enseñanza se añaden a la lista desbordada del paro, se producen manifestaciones en las calles y las mismas noticias con carácter formal se afirman y desmienten en canales formales.

El escenario estaba ya componiéndose con un atrezo que invitaba, en algunos casos, a una cierta incertidumbre de cambios antes de 2011 (Por ejemplo el 20 de mayo de 2010 se aprueba una rebaja entre el 0,56 y el $7 \%$ del salario a los funcionarios) pero el estudio 
realizado, especialmente a partir de mayo, se instala rodeado de una serie de acontecimientos que convierten al rumor en el apuntador de algunos de los actores principales de la enseñanza, los docentes. Entre otros, algunos de estos hechos en 2011 han sido:

- El 20 de septiembre se produce la primera huelga en la enseñanza, el 29 de noviembre, pese al desgaste, se alcanza la novena jornada contra los "recortes educativos" en la región. A Secundaria se unían esta vez Primaria, Infantil y estudiantes universitarios que se manifestaban en diferentes puntos de la capital (Álvarez, 2011a).

- El 20 de noviembre se celebran elecciones generales, muchos cambios quedan en el aire, al menos hasta la señalada fecha, algunos profesores fueron a votar con la camiseta verde; color este que simboliza la protesta y que junto a pancartas se pueden ver en muchos de los centros públicos durante meses (Álvarez, 2011b).

- En noviembre, dos días antes de las Elecciones Generales, se aprueban los nuevos temarios para oposiciones; en febrero del 2012, con el nuevo Gobierno, se suprimen estos cambios (Planelles y Alcaide, 2012). Fuera ya de los contratiempos provocados, tanto por la aprobación como por la supresión de la medida, y que afecta no solo a interinos o estudiantes de oposición, sino a otros colectivos como academias y editoriales, existe la sensación de que las noticias oficiales pierden su peso en cuanto a confianza, dando la razón a predicciones populares, aunque solo sean casuales, que coinciden con la últimas informaciones.

- En 2012 la oferta de plazas para docentes en Madrid, tras la sospecha de que no hubiera, fue de 190 solo para secundaria, no se convocaron puestos de Profesores Técnicos de FP. En 2010 hubo 1.425, con 120 para FP, en esta anterior convocatoria (recuérdese que es cada dos años) la oferta docente para toda España fue de 16.000 plazas frente a las 3.095 de 2012 (Álvarez, 2012).

Posterior a la recogida de cuestionarios y entrevistas en profundidad, en mayo de 2012 se siguen produciendo acontecimientos relacionados con la enseñanza en ocasiones tan consecutivos como cuando el 22 de mayo se convoca una huelga en todos los niveles educativos para llamar una vez más la atención sobre la escuela pública, recortes y reformas educativas, y un día después durante el Consejo de Universidades, los rectores no se presentaron al encuentro con el ministro de Educación como protesta a las medidas sobre tasa de matriculación y carga lectiva de los profesores (Aunión, 2012).

Los datos sobre manifestantes o asistentes a una huelga, según quien los aporte, muestran unas diferencias que llegan a modificar el titular de una noticia y, a veces, su intensidad cuando aparecen en medios oficiales como prensa o TV. Por alguna razón los muchos medios de comunicación de esta sociedad de la información tiene conocimientos o desconocimientos- muy diferentes ante la misma realidad; algo tan objetivo como una cifra de participación es zarandeada al capricho de los intereses particulares, esto no hace sino incrementar las posibilidades de rumorear sobre cuál será el poseedor de la verdad, cada opinión puede respaldarse en lo expuesto en la información y los canales formales que se acerquen más a su postura.

En ocasiones el rumor se puede transmitir sabiendo de su veracidad o conociendo su falta de exactitud pero, ¿por qué mentimos? La mentira puede verse como elemento que forma una garantía de la no existencia de límites, ante un mismo hecho suelen ser más las verdades referidas a él que las argumentaciones contrarias o diferentes del suceso, pero son incluso los límites, necesarios para la construcción de relatos no verdaderos, pues es sabido que el buen mentiroso habla poco (Mendiola, 2006:166). Estas informaciones, en principio, no se analizan como los datos oficialmente publicados, pero somos conscientes 
de que existen, sería un error tanto atribuirlos veracidad absoluta por cualquier parte afectada o participarte como no desmentirlo, en caso de su falsedad, por parte sobretodo de la cúpula organizativa (Díaz y García, 2011:361-363). Determinados mitos, a veces no solo del pasado, se sostienen sobre grandes grupos a pesar de su falsedad debido en parte a desconocimiento de la materia, otras por la teoría de la disonancia cognitiva de Festinger o por una manipulación interesada (Palacios, 2007:168-173); sin embargo en cuanto a los rumores en la enseñanza estamos hablando de pensamientos actuales y donde el grupo o red donde circula, los profesores, conocen las temáticas e intuyen las consecuencias de los cambios o decisiones. En momentos de crisis las comunicaciones se ven afectadas por dos factores: la velocidad con la que surge la información y el menoscabo que sufre la misma fruto de un entorno pesimista (Piñuel, 1997:171). En una época donde, en ocasiones, los errores se encadenan a justificaciones increíbles, son los rumores los que se liberan entre un público que busca el porqué de lo sucedido, a veces premeditados, pero otras espontáneos y exentos de correcciones, ideologías o acercamientos a otras causas. Como en cualquier otro sector laboral, en la enseñanza, siempre habían existido cambios, diversidad de opiniones y porque no, puede que un grado de incertidumbre que rozaba el pesimismo, pero los últimos acontecimientos, independientemente de los gobernantes, han doblado de nuevo el alambre del ánimo.

\section{LOS PROFESORES TÉCNICOS DE FORMACIÓN PROFESIONAL}

Son los profesionales que imparten clase a los Ciclos Formativos de Grado Medio y Superior de Formación Profesional, tienen como requisitos a la hora de presentarse en la oposición poseer un título de Diplomado Universitario, Arquitecto Técnico o Ingeniero Técnico o acreditar dos años de experiencia en la Comunidad de Madrid junto con la posesión de titulaciones de Técnico Especialista o superior en una especialidad de FP. Los ciclos de grado medio y superior son también impartidos por profesores de otras especialidades diferentes a las de profesores técnicos de FP, como por ejemplo FOL (Formación y Orientación Laboral).

Ha sido frecuente escuchar críticas hacia los profesores, de manera genérica, en base a sus horas de trabajo o periodos de vacaciones, conviene recordar que esta profesión implica, no sólo las horas de impartición de clases, sino otras como preparación de la materia, corrección de exámenes y ejercicios, tutorías, reuniones de departamento o claustros, tema diferente es que cada uno cumpla con sus obligaciones. Los profesores de secundaria y FP, grupo A nivel 24, además tienen menor sueldo que el resto de grupos de la Administración, argumentándolo en las vacaciones suplementarias del mes de julio con las que cuentan, siendo este mes de libre disposición por el centro y el Área Territorial. Además se está hablando de puestos a los que puede acceder todo el público con la titulación y requisitos exigidos por oposición y compitiendo en un proceso selectivo.

En el ámbito nacional, la evolución del profesorado técnico de FP para los cursos 200001, 2005-06, 2009-10, 2010-2011 ha sido respectivamente de 21.044, 20.886, 23.958 y 24.278 según los datos de las plantillas presupuestarias. Frente a un alumnado matriculado en 2000-2001, 2005-06 y 2010-2011 de 449.008, 454.053 y 578.574 respectivamente. En la Comunidad de Madrid durante el curso 2010-2011 el total de alumnos matriculados en Ciclos Formativos de Grado Medio y Superior (tanto en la modalidad presencial como a 
distancia) fueron de 63.448 alumnos según se recoge en los Datos y Cifras del Curso Escolar 2011/2012 del Ministerio de Educación.

Existen dos grandes grupos de profesionales: funcionarios e interinos, (con situaciones administrativas distintas) estos últimos son aquellos docentes que no han obtenido plaza, (pese a poder haber aprobado, y con buena nota, los procesos selectivos, en contra de lo que se ha manifestado en algunos medios) y que se encuentran ordenados por lista a la espera de ser llamados para cubrir una sustitución. Hay que señalar que basta una sola negativa para estar excluido de la lista salvo causas de renuncia justificadas. Muchos de los trabajadores interinos lo son durante años, el número en Madrid de afectados por las últimas medidas supera los 3.000, entre ellos se encuentran profesores, ahora desempleados, que habían prestado servicio de manera ininterrumpida durante más de 10 años (Barcala, 2011).

\section{INSTRUMENTOS UTILIZADOS EN EL ESTUDIO}

Para su confección y antes del definitivo cuestionario se puso en práctica un pretest en el que probar aceptación de las preguntas, interpretación correcta y funcionamiento del método; confirmado su validez, y comenzando por la puesta en conocimiento del director o responsable de cada uno de los centros públicos con FP de la DAT Sur del proyecto de investigación, se procedió a entregar los cuestionarios. Una vez en el centro se repartieron no solo a los profesores de FP, sino al resto de docentes y profesionales que trabajan en los institutos, las fuentes y los transmisores del rumor no están sujetas a escalas jerárquicas, departamentos o protocolos formales. 332 cuestionarios fueron realizados en los 27 centros que accedieron de un total de 45 . El cuestionario constó de 43 ítems, precedidos de una hoja de presentación del proyecto. En la mayoría de las preguntas se utilizó una escala likert con 5 opciones.

Se trató de marcar la diferencia entre las respuestas aportadas por los diferentes grados de experiencia en el centro, tipo de trabajador (interino o funcionario), así como entre quienes tienen o no personas a su cargo. Directores, jefes de departamento o jefes de estudio pueden tener acceso de manera diferente tanto a fuentes formales como informales por las características de su puesto. Un objetivo fue conocer en qué medida y con qué diferencia proporciona más motivación para trabajar la información informal o la formal. Interesaba saber cómo afecta el estado de ánimo de los compañeros sobre el encuestado, y sobre la influencia del clima laboral en el departamento donde se trabaja. Una incógnita planteada fue averiguar el espacio de tiempo en el que la comunicación informal aporta más noticias, es decir en qué momento del día o de la jornada laboral. Fue sugerente intentar demostrar el medio para transmitir esa información, así como de localizar en qué lugar del trabajo la comunicación informal aporta más datos. Se buscó conocer en qué grado los rumores tienen fiabilidad, si la información que aportan es comparable a la de un medio oficial no solo en cuanto al detalle sino en cuanto a anticiparse a la formal; surge la duda en cuanto al rumor saber en qué medida se difunde a otros compañeros y si se comparan ante un mismo hecho las fuentes formales e informales.

Como es lógico en conversaciones o reuniones informales dentro del centro no se habla únicamente de temas relacionados con el trabajo, se intentó medir cuando se realizan conversaciones atendiendo a los horarios laborales con otros trabajadores tanto de temas laborales como de otros. El día de la semana puede influir en el estado de ánimo, la motivación y también en los momentos preferidos para hacer comentarios con compañeros, 
los encuestados tuvieron la opción a elegir el día o los días de la semana más señalados para ello.

En cuanto a las entrevistas en profundidad, fueron realizadas también en el propio centro y respetando de nuevo el anonimato, el objetivo era cruzar y confirmar algunos de los resultados obtenidos por el cuestionario y recoger información de manera más amplia sobre las cuestiones planteadas. Realizadas personalmente, previa cita telefónica con el director o jefe de departamento de los IES, en algún caso por motivos de incompatibilidad horaria se rellenaron de forma escrita. Se completaron 17 en los 4 centros elegidos por su mayor y menor participación en los cuestionarios y alternando entre puestos diferentes como: director, jefe de departamento, docente de FP, docente en otras enseñanzas y personal no docente. En el resultado del cuestionario se marca que el "cara a cara" es mucho más utilizado para transmitir rumores que por ejemplo los chats, el móvil u otros medios. Había que indagar cual era la razón, para intentar ver posibles opciones de medir o controlar los rumores aun en su forma más tradicional.

En varios encuentros con docentes se afirmó que los profesores de primaria tenían más conversaciones con alumnos y compañeros fuera de las clases, por lo que surge averiguar si en secundaria hay menos rumores y sobre todo si puede atisbarse alguna diferencia de FP con otras enseñanzas. Existe la hipótesis pendiente de confirmar que academias, sindicatos y partidos políticos son en algunos casos beneficiarios de rumores en la enseñanza, especialmente respecto a las fechas de oposición, baremaciones, exámenes o todo lo relacionado con tiempo y/o remuneración vinculados con el trabajo. Algunos directores o jefes de estudios han hecho comentarios respecto a los motivos que llevan a no rellenar e incluso negarse a rellenar cuestionarios, fue un propósito averiguar el motivo de forma más amplia y directa. El tiempo medio de las entrevistas fue de 20 minutos transcurridos en la mayoría de los casos en los propios despachos de los encuestados, sala de profesores e incluso los pasillos.

\section{ANÁLISIS E INTERPRETACIÓN DE LOS RESULTADOS}

La proporción de hombres y mujeres obtenida es de $63,2 \%$ para estas últimas, coincidiendo con la media de profesoras en todos los niveles educativos que facilitan los datos oficiales obtenidos durante el 2011 y que llegan al 64\%. En FP este porcentaje es bastante inferior, pero el estudio se ha hecho a todos los integrantes que trabajan en los centros de enseñanza. Por edades, la máximas horquillas obtenidas están entre los 40 y 50 años, con un $42 \%$ y los que superan los 50 con un $31,9 \%$, la suma el $73,9 \%$ de los que superan los 40 años coincide con el $72,4 \%$ que aporta el ministerio a nivel nacional en los Datos y cifras del curso escolar 2011/2012.

Se entiende que la información, tanto formal como informal, provoca motivación a la hora de trabajar y en muchos casos la atracción por conocer rumores podría alentar esa motivación, sin embargo es la información oficial la que proporciona mayor motivación para trabajar en el estudio realizado, tanto para jefes como para subordinados, teniendo en cuenta que solo un 34\% tienen personas a su cargo (por ser directores, jefe de departamento, etc.). Los encuestados opinan que su estado de ánimo contagia al resto de compañeros en la misma medida que todo el grupo puede influir en el suyo, pero señalan la importancia del clima 
general del centro sobre sus departamentos, incluso aunque en algunos casos lo formen grupos de pocas personas.

Pese a la llegada de las nuevas tecnologías la conversación "cara a cara" sigue siendo la forma más practicada para hacer comentarios, muy por encima de la utilización del móvil o Internet. Se preguntó sobre esta práctica en la entrevista obteniendo que ello era debido a las siguientes causas: tenemos una necesidad de que respalden lo que decimos, que se produce instantáneamente cuando vemos la cara de nuestros oyentes particulares, además lo que afecta personalmente se prefiere transmitir personalmente, Internet es más usado para contar lo que afecta como grupo, en una conversación "cara a cara" no hay que preparar nada, es totalmente espontánea, seguimos valorando el contacto humano y hay un componente de edad que todavía no ha llegado a los mayores de 45. Existe además un grado de confianza (de más o de menos) que aventaja a las nuevas tecnologías: si no tenemos la suficiente confianza para conocer el número de teléfono o dirección de correo haremos el comentario "cara a cara", por otro lado si tenemos un alto grado de confianza nos será más agradable también contarlo personalmente, los móviles y el correo electrónico estarían en el medio o servirían de apoyo. Es interesante comprobar que Internet es mucho más usado que el móvil, cuadruplicando la aportación de noticias con carácter informal (las no oficiales).

En cuanto al lugar del centro donde se produce mayor divulgación de rumores es en la sala de profesores, seguido del comedor junto a la cafetería. El origen de los mismos en el centro de trabajo obtuvo los siguientes resultados: un 12,3\% los atribuye a la Consejería de Educación, un 3,4\% opina que surgen de la dirección del propio centro, y un 68,4\% considera que surgen de sus propios compañeros. En la entrevista en profundidad, sin embargo la opinión variaba, se situaba a la Consejería como lanzadora de rumores y maniobrar con datos oficiales, siendo los profesores simples transportistas de los comentarios de la Consejería y de la dirección del centro, también se afirmó que suelen ir contra alguien y en base a ello son originados por uno u otro sector. El 70\% de los encuestados contrasta la información con la que les comunican los medios oficiales, a pesar de ello, más de un $25 \%$ de todos los encuestados opinaron que una misma información les llega antes de manera informal que por medios oficiales.

Además de los compañeros de profesión, otras personas se convierten en facilitadores de noticias relacionadas con el trabajo, en este orden de importancia: conocidos/amigos docentes ajenos al centro, alumnos, personal de admón. y servicios (PAS) y padres o familiares de alumnos. Cada profesional mantiene conversaciones referidas al trabajo de manera informal con grupos de compañeros cercanos a los 7 individuos en el centro, e inferiores a 5 fuera del mismo, se estima que de los primeros unen las relaciones/condiciones laborales y en segundo término la edad más que otras como podrían ser cercanía del domicilio o experiencia que se dan más en otros sectores de trabajo distintos a la enseñanza, aunque destaca la simpatía política frente a los acontecimientos (como huelgas u otras formas de protesta) por encima de otras variables a la hora de elegir amistades. Es el inicio y fin de semana laboral (lunes y viernes) los días preferidos por la mayoría para hacer comentarios a sus compañeros incluso más que la suma de los restantes (martes, miércoles, jueves, sábado y domingo). Influye aquí el hecho, ya descrito, que se haga principalmente "cara a cara", además las novedades se comentan tras el fin de semana y la motivación que existente el viernes a hacer comentarios es debido a factores como: celeridad por transmitir información antes dar comienzo la otra semana, hiperactividad social de la antesala de los días no laborales y noticias propias o producidas ese día. 
Contrariamente a lo que ocurre en otros sectores, en la enseñanza, espacios y momentos como: de camino al trabajo o al salir de este, pasillos o incluso la cafetería no son utilizados con preferencia para hacer comentarios. La sala de profesores es preferida para ser usada un $40 \%$ más que la cafetería para aportar noticias de carácter informal. También otras diferencias con sectores distintos a la enseñanza se encuentran en espacios como pasillos o incluso aseos por funcionar como bypass entre tiempos de trabajo, los profesores sin embargo al tener entre ellos clases y horarios diferentes coinciden menos en estos lugares. La preferencia para hacer un comentario es durante la mañana en un $62 \%$. Solo un $8 \%$ afirman merecerles fiabilidad sus contenidos, sin embargo un $24,8 \%$ aseguran que una misma información les llega antes a través de los rumores que de forma oficial. Pese a que muchas noticias de carácter oficial sorprenden a todos y estaban fuera de cualquier hipótesis, se indica que en la mayoría de los casos teniendo en cuenta que los rumores se mueven en todas direcciones, preceden a cualquier cambio oficial, el problema viene por identificar cual es el correcto, de hecho más del $60 \%$ contrasta la comunicación informal con la formal (oficial).

Se pudo comprobar también que en muchos casos no se rellenan o hay una negación a rellenar cuestionarios; existen varios factores como, falta de tiempo, exceso de burocracia, o que muchas otras investigaciones piden su colaboración, (incluso los propios alumnos manifiestan dudar de lo que puede cambiar las valoraciones que rellenan) a veces simple pereza, una excesiva utilización comercial de los centros, un bombardeo constante para pedir o dar información ajena a la enseñanza que produce agotamiento, pero sobre todo existe una desmotivación por pensar que toda participación que se salga de la rutina del trabajo no servirá para nada, hay un desinterés colectivo; hay también una desconfianza a dar información privada pese a tratarse de cuestionarios anónimos y un enfado general de cualquier cambio que proceda de la Administración. Existe la sensación de que a los profesores se les exige una vocación pero a la que no acompaña un reconocimiento de su trabajo.

Ante la pregunta de cómo controlar los rumores en los centros de enseñanza, partiendo de la base de que es tarea complicada, la mayoría si recomienda que la información sea periódica tanto por parte del centro como por la Administración, destaca como primer objetivo escuchar (conocer la existencia del rumor) y como segundo conocer su origen; otra estrategia, una vez detectado, es tratar de reunir a los principales implicados (en el caso de ser profesionales del centro) y formalmente exponer la información oficial si se conoce. Parecen estar todos de acuerdo en que nadie se beneficia de ellos, en el sentido práctico de recibir la información; si hay por el contrario una sospecha, de quienes los conocen, de sentirse privilegiados de poseer esa información. En ocasiones se trata de un simple desahogo por quien los cuenta y una satisfacción de curiosidad de quien los oye. El rumor beneficia al propio rumor, su puesta en práctica, aunque sea falso, le da la sospecha de credibilidad para permanecer en el tiempo en función de cómo se difunda.

\section{CONCLUSIÓN}

En esta comunicación entre la Administración, el centro y los profesores, un pensamiento reflejado por muchos de estos últimos era plantearse la pregunta ¿para cuándo una ley de educación que escuche a los docentes? Con las actuales modificaciones esta preocupación pasa a un segundo plano relevada por las propias condiciones laborales. Los rumores en 
algunos casos ponen en estado de alerta, asustan incluso, pero los profesores están cansados de cambios e incertidumbres, como profesión es vinculada a una alta tasa de ansiedad, reconocida socialmente, pero no recompensada ni emocional ni económicamente sumado a un deseado aumento de prestigio social observado en otros países.

A la hora de buscar su origen en la enseñanza se puede afirmar que: vienen provocados por diferencias de opiniones, esto unido a la forma de ser humana de querer contar y querer oír (interrelación); y las agrupaciones de pensamientos y grupos nacen también de las grietas que tiene la comunicación formal como son: la posibilidad de ser lenta, incompleta, poco argumentada (creer que la noticia no es cierta), variable (cambiando en el corto y medio plazo) o injusta (algo muy subjetivo). En una tormenta de noticias formales, e informales sobre todo son los sindicatos y las academias los paraguas donde refugiarse en un primer momento, pero afectados junto a los docentes, y no beneficiados ni de las medidas expuestas en medios oficiales que inquietan a la enseñanza, ni de su difusión formal o informal.

La incertidumbre produce que se originen y se difundan rumores, al tiempo, estos mismos por sí solos, formando no solo un ciclo que se retroalimenta, sino a veces, una espiral, provocan de nuevo la formación de incertidumbre, pero esta se ve reducida, aunque sea levemente, cuando quien nos escucha afirma lo que decimos. La ilusión es la fabricación artificial de un tiempo de espera positivo y optimista que calma esta duda ansiosa.

En ocasiones sirven para contar lo que la organización no se atreve a decir, o para probar como actuaría el grupo o la sociedad si la noticia fuera confirmada por medios oficiales. Su censura no es ni siquiera acotada por la credibilidad de quien lo escucha, pues a veces podemos contar historias que nos contaron y que ni nosotros podemos probar con tal de ser escuchados y de provocar emociones a nuestros próximos oyentes.

Este tipo de información cuenta con la desventaja de ser acusada de su falta de certeza incluso antes de ser comprobada, pero aventaja a las noticias formales en que el sentimiento prolongado de esperanza o miedo de que algo se cumpla es mayor que el provocado por la certeza, sea esta buena o negativa Para quien estudia una oposición tener durante un periodo una duda razonable de que se van a convocar plazas puede acumular más pasión que conocer cierta y afirmativamente la noticia. Los rumores son esos mensajes que nos gustaría oír cuando los escuchamos y que intentamos les agraden a los que se los contamos.

Pero, ¿qué ocurre con los mensajes pesimistas? Pensar que se puede tener la información, aunque sea negativa, es mejor que no tener ninguna, más incertidumbre que provoca el rumor, a falta de datos oficiales, es la que habita en un espacio donde ni siquiera hay rumores. Son como una llama que sirve desde, para quemar la construcción de pensamientos, hasta para hacer viajar un sueño olímpico, pero con la que siempre alumbraremos dos ilusiones: la de quien lo cuenta y quien lo escucha.

\section{BIBLIOGRAFÍA}

ÁLVAREZ, P. (2011a), "La participación en la novena jornada de huelga en la Enseñanza cae hasta un 29,4\%" en http://elpais.com/elpais/2011/11/29/actualidad/, visitado 02/06/2012.

ÁLVAREZ, P. (2011b), "Veto electoral a la camiseta "verde" en http://elpais.com/diario/2011/11/28 /madrid/1322 483058_850215.html, visitado 02/06/2012.

ÁLVAREZ, P. (2012), "Madrid quiere ofertar 150 plazas de oposiciones a enseñanza secundaria" en http://www.que.es/ultimas-noticias/sociedad/201202191010-opositores-deberan-conformarse-3095plazas-efe.htm, visitado 02/06/2012. 
AUNION, J. A. (2012), "Los rectores rechazan el endurecimiento de los requisitos para obtener becas" en http://sociedad.elpais.com/sociedad/2012/06/01/actualidad/1338535992_317758.html, visitado 10/06/2012.

BARCALA, D. (2011), "Las cifras avalan que hay 3.000 docentes menos" en http://www.publico.es /espana/399257/las-cifras-avalan-que-hay-3-000-docentes-menos, visitado 10/06/2012

BARKER, A. (2001), Como mejorar la comunicación. Barcelona: Gedisa.

BEL, J. I. (2005), Comunicar para crear valor. Navarra: EUNSA.

BURKE, P. (2011), "El poder de los rumores", en ABC Cultural, 1 octubre, pp. 18-19.

DÍAZ CANO, E. y GARCÍA MANSO. A. (2011), "La comunicación en momentos de crisis empresariales: estrategias 2.0.", en Innovaciones en la sociedad de riesgo, ACMS, pp. 349-370.

FERNANDEZ, C. (2003), La comunicación en las organizaciones, Méjico, Trillas.

GALLEGO, S. (2011), "Redes sociales y desarrollo humano", en Barataria. Revista CatellanoManchega de Ciencias Sociales, $\mathrm{n}^{\mathrm{O}}$ 12, pp. 113-121.

GARCÍA MANSO, A. y DÍAZ CANO, E. (2010), "Estrategias de Comunicación empresarial” en Construcciones y deconstrucciones de la sociedad, ACMS, pp. 307-220.

LUCAS, A. (1997), La comunicación en la empresa y en las organizaciones. Barcelona: Bosch.

MARTINEZ DE VELASCO, A. y NOSNIK, A. (2006), Comunicación organizacional práctica. Méjico: Trillas.

MAZO, M.E. (1996), El rumor y su influencia en la cultura de las organizaciones. Madrid: UCM.

MENDIOLA, I. (2006), Elogio de la mentira en torno a una sociología de la mendacidad. Madrid: Lengua de Trapo.

MINISTERIO DE EDUCACIÓN. SECRETARIA GENERAL TÉCNICA. (2011), "Datos y cifras Curso escolar 2011/2012" en http://www.educacion.gob.es/horizontales/estadisticas/indicadorespublicaciones-sintesis/datos-cifras.html, visitado 02/06/2012.

O'C LEGGETT, B. (1993), Comunicación oral en la empresa. Navarra: Eunsa.

OLIVER, D. (2005), Cómo negociar eficazmente. Barcelona: Gedisa.

PALACIOS, J.L. (2007), "Hernán Cortés según Neil Young: Una aproximación desde la sociología del conocimiento a un caso paradigmático de distorsión cognitiva", en Barataria. Revista CatellanoManchega de Ciencias Sociales, $\mathrm{n}^{\circ}$ 8, pp. 161-179.

PIÑUEL, J.L. (1997), Teoría de la comunicación y gestión de las organizaciones. Madrid: Síntesis.

PLANELLES, M. y ALCAIDE, S. (2012), "Wert niega que el cambio de temarios sea "dañino" para los opositores" en http://sociedad.elpais.com/sociedad/2012/02/08/actualidad/1328699513_883318. html, visitado 02/06/2012.

SAAVEDRA, I. (1998), Motivación y comunicación en las relaciones laborales, Madrid, Pirámide. UÑA, O. (1994), Comunicación y libertad: la comunicación en el pensamiento de Karl Jaspers. Madrid: Ediciones Escurialenses, D.L.

\section{Breve currículo:}

\section{Francisco José Ramiro de la Mata}

Licenciado en Ciencias del Trabajo y Graduado Social por la Universidad Rey Juan Carlos. Master Oficial en Gestión e Investigación de la Comunicación Empresarial. Docente en Formación de Adultos y en Ciclos Formativos de Grado Medio, tanto en centros públicos como concertados. Su línea de investigación es la comunicación en el ámbito laboral sobre lo que ha publicado diferentes artículos. Cuenta con experiencia en el plano empresarial en diversos departamentos: Clientes, Recursos Humanos y Contabilidad. 